\title{
Magnetic and ferroelectric characteristics of $\mathrm{Gd}^{3+}$ and $\mathrm{Ti}^{4+}$ co-doped $\mathrm{BiFeO}_{3}$ ceramics
}

\author{
SHIVANAND MADOLAPPA, A V ANUPAMA, P W JASCHIN, K B R VARMA and B SAHOO* \\ Materials Research Centre, Indian Institute of Science, Bangalore 560012, India
}

MS received 19 October 2015; accepted 29 October 2015

\begin{abstract}
Polycrystalline $\mathrm{BiFeO}_{3}$ and $\mathrm{Bi}_{0.9} \mathrm{Gd}_{0.1} \mathrm{Fe}_{1-x} \mathrm{Ti}_{x} \mathrm{O}_{3}(x=0,0.01,0.05$ and 0.1$)$ samples were synthesized by solid-state reaction route. Structural, magnetic and ferroelectric properties of these samples were investigated. $\mathrm{X}$-ray powder diffraction (XRD) results confirmed the presence of a significant amount of $\mathrm{Bi}_{2} \mathrm{Fe}_{4} \mathrm{O}_{9}$ impurity phase in the undoped $\mathrm{BiFeO}_{3}$ sample. Mössbauer spectroscopy studies corroborated the XRD studies to confirm the presence of impurity phase. We have observed that gadolinium $\left(\mathrm{Gd}^{3+}\right)$ and titanium $\left(\mathrm{Ti}^{4+}\right)$ doping, respectively, on $\mathrm{Bi}^{3+}$ and $\mathrm{Fe}^{3+}$ sites facilitated a significant reduction in the impurity phase formation in $\mathrm{BiFeO}_{3}$. Interestingly, $\mathrm{Gd}^{3+}$. doping significantly reduced the impurity phase formation as compared to the undoped $\mathrm{BiFeO}_{3}$ sample. This impurity phase formation was further overcome by doping higher $(x \geq 0.05)$ amounts of $\mathrm{Ti}$ in $\mathrm{BiFeO}_{3}$. The crystallographic site occupancies of Gd and Ti were confirmed by Rietveld refinement of XRD data, Mössbauer spectroscopy and magnetization measurements. An enhancement in ferromagnetic properties along with moderate ferroelectric properties have been observed after co-doping. There was an increasing trend in remnant polarization $\left(P_{\mathbf{r}}\right)$ with the increase in Ti concentration besides an improvement in the characteristic saturation magnetization. Our results demonstrate that $\mathrm{Gd}^{3+}$ and $\mathrm{Ti}^{4+}$ doping could be used to enhance multifunctional properties of $\mathrm{BiFeO}_{3}$ ceramics to enable them as potential material for various devices.
\end{abstract}

Keywords. Multiferroic; bismuth ferrite; Rietveld analysis; Mössbauer spectroscopy.

\section{Introduction}

Multiferroic materials are in increasing demand for a wide range of applications that include information storage, spintronic devices, sensors, electric field controlled magnetic devices and multiple state memory elements, etc [1-4]. The existence of multiferroic materials is obscure in nature, because they need to simultaneously possess empty as well as partially-filled transition metal orbitals to exhibit ferroelectric and ferromagnetic properties, respectively $[5,6]$. Bismuth ferrite (BFO) is one such material in which multiferroic characteristics have been realized. BFO has a rhombohedrally distorted perovskite structure at room temperature associated with the space group $R 3 c$. It has both ferroelectric $\left(T_{\mathrm{c}} \sim 1103 \mathrm{~K}\right)$ and G-type antiferromagnetic $\left(T_{\mathrm{N}} \sim 643 \mathrm{~K}\right)$ ordering (with a cycloidal spin modulation period of 620 A) [7]. The ferroelectricity in BFO originates from the lone electron pair $\left(6 \mathrm{~s}^{2}\right)$ of $\mathrm{Bi}^{3+}$ ions and the antiferromagnetic property is due to the presence of $\mathrm{Fe}^{3+}$ ions [8]. However, high leakage current associated with BFO limits its applications. As a result, it is very difficult to demonstrate true ferroelectric behaviour of BFO as it does not exhibit typical polarization vs. electric field hysteresis loops owing to the low resistivity associated with the samples [9].

\footnotetext{
*Author for correspondence (bsahoo@mrc.iisc.ernet.in)
}

One of the sources of high leakage current is the impurity phases that form during synthesis of BFO. It has been observed that the enthalpy of formation of BFO $\left(768 \mathrm{~kJ} \mathrm{~mol}^{-1}\right)$ is much larger than that of $\mathrm{Bi}_{2} \mathrm{Fe}_{4} \mathrm{O}_{9}\left(-2276 \mathrm{~kJ} \mathrm{~mol}^{-1}\right)$ and $\mathrm{Bi}_{25} \mathrm{FeO}_{39}\left(-7699.8 \mathrm{~kJ} \mathrm{~mol}^{-1}\right)$, leading to easy formation of these undesired impurity phases [10]. Also the volatility of $\mathrm{Bi}_{2} \mathrm{O}_{3}$ phase to some extent results in the formation of the bismuth-deficient impurity phases.

It was suggested in the literature that the ferroelectric and magnetic properties of BFO ceramic could be improved by doping with suitable ions [11], which breaks the cycloidal antiferromagnetic structure [12]. Choice of proper dopants and their concentrations in BFO have been challenging in terms of obtaining phase pure BFO. Such doped samples often show large magneto-electric coupling manifested by huge magnetic and ferroelectric responses. For instance, rare-earth ion doping at $\mathrm{Bi}$ site in $\mathrm{BFO}$ was found to minimize the impurity phase formation and resulted in improving the magnetization by suppressing antiferromagnetism and its cycloidal spin structure $[13,14]$ besides an enhanced ferroelectric property. Also, BFO with transition metal ion doping has been evidenced to be very effective in improving their insulating and magnetic properties $[15,16]$.

Large effective magnetic moment $\left(8.0 \mu_{\mathrm{B}}\right)$ associated with $\mathrm{Gd}^{3+}$ ions encouraged us to consider $\mathrm{Gd}$ as a potential dopant for the Bi-site in BFO to enhance the magnetic properties [17]. On the other hand, $\mathrm{Ti}^{4+}$ was selected as potential donor dopant on $\mathrm{Fe}^{3+}$ site. This choice is due to the polarizing 
nature of $\mathrm{Ti}$ similar to that in the case of $\mathrm{BaTiO}_{3}$ [18]. As a consequence, it is expected to improve the ferroelectric property by reducing electrical conductivity. In this work, we have synthesized undoped and doped $\mathrm{BFO}$, i.e., $\mathrm{BiFeO}_{3}$ and $\mathrm{Bi}_{0.9} \mathrm{Gd}_{0.1} \mathrm{Fe}_{1-x} \mathrm{Ti}_{x} \mathrm{O}_{3}(x=0,0.01,0.05$ and 0.1$)$ samples by the solid-state reaction route. Here we report the details of our investigations into the structural properties (by Rietveld refinement) and Mössbauer spectroscopy of these compounds besides their ferroelectric and magnetic properties. Though we carried out the dielectric property study as a function of dopant concentration in the wide range of frequencies, our observations are similar to those observed by Basith et al [19] and Manoj et al [20] and therefore, these studies are not included. The $\mathrm{Bi}_{0.9} \mathrm{Gd}_{0.1} \mathrm{Fe}_{1-x} \mathrm{Ti}_{x} \mathrm{O}_{3}$ samples for $x=0,0.01,0.05$ and 0.1 are henceforth designated as BGF, BGFT1, BGFT2 and BGFT3, respectively.

\section{Experimental}

BFO, BGF, BGFT1, BGFT2 and BGFT3 samples were synthesized via the solid-state reaction route, using $\mathrm{Bi}_{2} \mathrm{O}_{3}$ (99.90\%, NICE), $\gamma-\mathrm{Fe}_{2} \mathrm{O}_{3}$ (99.00\%, NICE), $\mathrm{Gd}_{2} \mathrm{O}_{3}(99.90 \%$, HIMEDIA) and $\mathrm{TiO}_{2}(99.90 \%$, MERCK) as starting materials. Prior to mixing, stoichiometric amounts of raw materials were weighed and ground by using agate mortar and pestle for $\sim 2 \mathrm{~h}$ in acetone medium. This mixture was calcined at $700^{\circ} \mathrm{C}$ for $5 \mathrm{~h}$. Thus, obtained powders were then mixed well with a small amount of poly-vinyl alcohol (PVA) as binder and pressed into pellets of $10 \mathrm{~mm}$ in diameter and $1.5 \mathrm{~mm}$ in thickness by applying a load of $40 \mathrm{kN} \mathrm{cm}^{-2}$ for about $2 \mathrm{~min}$. The BFO pellet was sintered at $800^{\circ} \mathrm{C}$ for $5 \mathrm{~h}$, whereas the BGF, BGFT1 and BGFT2 pellets were sintered at $850^{\circ} \mathrm{C}$ for $5 \mathrm{~h}$. However, the BGFT3 pellets were not mechanically stable and were re-sintered at $925^{\circ} \mathrm{C}$ for $5 \mathrm{~h}$ in air. The experimental density was determined by Archimedes method. For ferroelectric measurements, the pellets were ground and polished to a typical thickness of $0.7-0.74 \mathrm{~mm}$ and silver electrodes were applied on major faces of the pellets. The pellets in two electrode configurations were dried at $150^{\circ} \mathrm{C}$ for $24 \mathrm{~h}$.

$\mathrm{X}$-ray diffraction (XRD), vibrating sample magnetometry (VSM) and Mössbauer spectroscopy (in transmission mode) studies were performed on the powders from crushed pellets. XRD patterns were collected using 'Philips PAN Analytical' diffractometer with $\mathrm{Cu}-\mathrm{K}_{\alpha}(1.54056 \AA)$ radiation in the $2 \theta$ range $10-70$ degrees. The Rietveld analysis of XRD data was carried out using the 'FULLPROF' package [21]. Mössbauer spectra of all the analysed samples were recorded at room temperature (RT) by using a Mössbauer spectrometer (SEE Co.,${ }^{57}$ Co source $(\sim 15 \mathrm{mCi})$ and constant acceleration Doppler-velocity-drive). The morphology of the synthesized samples was studied by scanning electron microscopy (SEM) (FEI Inspect F50). The ferroelectric property was studied at RT by using Radiant Precision work station. Magnetic properties were studied at RT using vibrating sample magnetometer (VSM) (Lakeshore 615 USA).

\section{Results and discussion}

\subsection{Structural investigations}

3.1a X-ray diffraction: Measured and calculated XRD intensity patterns after Rietveld refinement for BFO, BGF, BGFT1, BGFT2 and BGFT3 samples are shown in figure 1. Rietveld refinement was performed on the XRD patterns of the entire series of samples under study to obtain the structural parameters precisely as given in table 1 . The major phases i.e., $\mathrm{BiFeO}_{3}$ and $\mathrm{Bi}_{0.9} \mathrm{Gd}_{0.1} \mathrm{Fe}_{1-x} \mathrm{Ti}_{x} \mathrm{O}_{3}$ have rhombohedral $R 3 c$ type crystal structure. In the following paragraphs the detailed phase analysis carried out for all the samples is illustrated.

According to the Rietveld refinement results, besides $82 \mathrm{wt} \%$ of $\mathrm{BiFeO}_{3}(R 3 c)$ phase, a significant amount of $\mathrm{Bi}_{2} \mathrm{Fe}_{4} \mathrm{O}_{9}(\sim 15 \mathrm{wt} \%)$ impurity phase is present along with a minute quantity of $\alpha-\mathrm{Fe}_{2} \mathrm{O}_{3}(R 3 c \sim 1 \mathrm{wt} \%)$ and $\mathrm{Bi}_{2} \mathrm{O}_{3}$ (Pccn $\sim 1 \mathrm{wt} \%)$ and orthorhombic $\mathrm{BiFeO}_{3}($ Pnma $\sim 1 \mathrm{wt} \%)$ in the $\mathrm{BFO}$ sample figure 1a. This unintended $\mathrm{Bi}_{2} \mathrm{Fe}_{4} \mathrm{O}_{9}$ phase has orthorhombic structure (Pbam). The lattice parameters of this impurity phase are: $a=7.9797 \AA, b=8.5490 \AA$, $c=6.0086 \AA$. The refinement results for lattice parameters

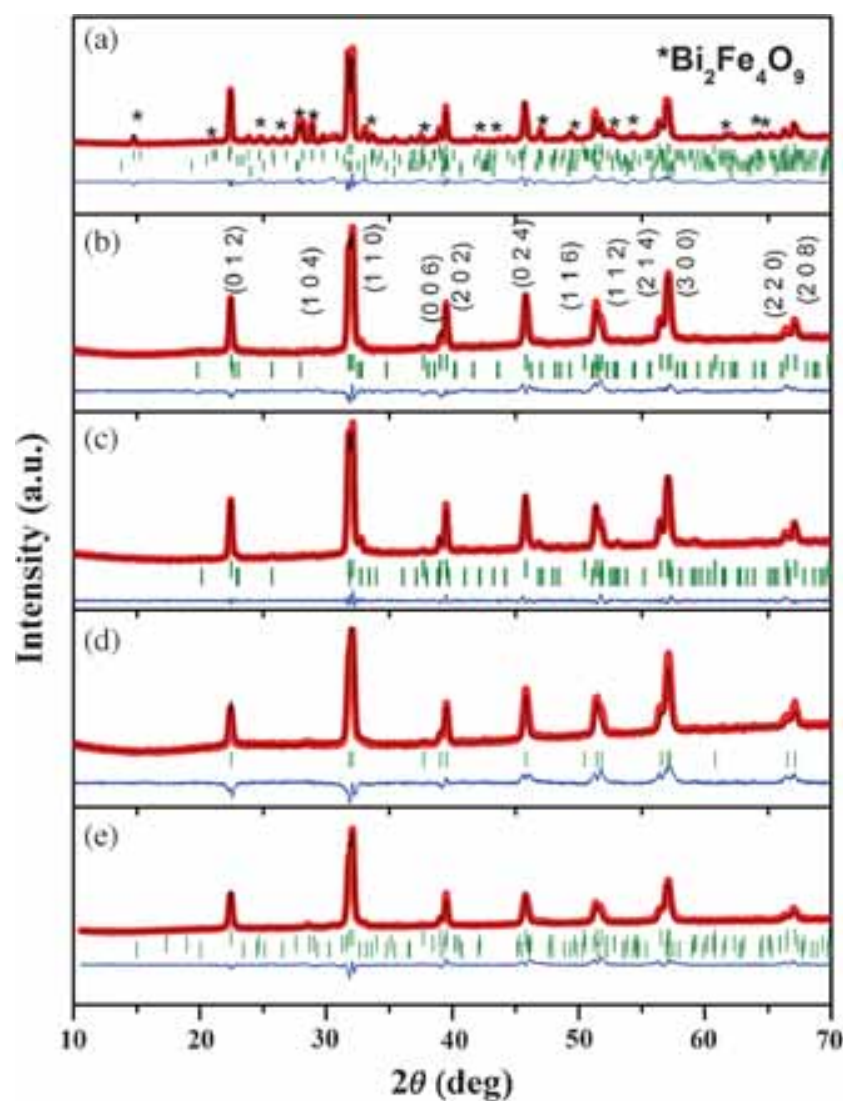

Figure 1. Rietveld refinement of the XRD patterns for (a) BFO, (b) BGF, (c) BGFT1, (d) BGFT2 and (e) BGFT3 samples at RT. The circles are the experimental data $\left(Y_{\exp }\right)$, the solid line is the calculated pattern $\left(Y_{\text {calc }}\right)$ and the bottom curve is the difference between the experimental and calculated patterns $\left(Y_{\exp }-Y_{\text {calc }}\right)$. 
Table 1. Rietveld refinement results of the XRD data for the samples. $R_{\mathrm{Bragg}}$ (Bragg factor), $\chi^{2}$ (Goodness of fit), $R_{\mathrm{f}}$ (structure factor), $R_{\mathrm{p}}$ (residuals for the unweighed pattern) and $R_{\mathrm{wp}}$ (residuals for the weighed pattern).

\begin{tabular}{lccccc}
\hline Parameters & BFO & BGF & BGFT1 & \multicolumn{1}{c}{ BGFT2 } & BGFT3 \\
\hline$a(=b)(\AA)$ & 5.5801 & 5.5764 & 5.5986 & 5.5905 & 5.5753 \\
$c(\AA)$ & 13.8717 & 13.8474 & 13.8513 & 13.8261 & 13.8314 \\
Volume $\left(\AA^{3}\right)$ & 374.064 & 372.909 & 373.277 & 371.549 & 372.336 \\
Strain $\times 10^{-3}$ & 3.15 & 3.55 & 4.12 & 4.62 & 3.68 \\
Crystallite size $(\mu \mathrm{m})$ & 0.84 & 0.40 & 0.46 & 0.49 & 0.18 \\
$R_{\text {Bragg }}$ & 4.82 & 3.06 & 2.93 & 7.03 & 7.80 \\
$R_{\mathrm{f}}$ & 3.85 & 2.71 & 2.94 & 11.2 & 7.92 \\
$\chi^{2}$ & 15.7 & 2.12 & 4.24 & 2.62 & 3.02 \\
$R_{\mathrm{p}}$ & 21.9 & 20.5 & 25.9 & 30.7 & 23.7 \\
$R_{\mathrm{wp}}$ & 17.3 & 11.6 & 15.7 & 15.6 & 14.1 \\
\hline
\end{tabular}

Table 2. Ideal elemental composition, phases present (in wt\%) obtained from XRD studies, elemental composition obtained from XRD studies, elemental composition obtained from EDAX studies, theoretical density $\left(\rho_{\text {theo }}\right)$, experimental density $\left(\rho_{\text {exp }}\right)$ and percent of densification for BFO, BGF, BGFT1, BGFT2 and BGFT3 samples.

\begin{tabular}{|c|c|c|c|c|c|}
\hline Parameters & $\mathrm{BFO}$ & BGF & BGFT1 & BGFT2 & BGFT3 \\
\hline $\begin{array}{l}\text { Ideal elemental } \\
\text { composition (at } \% \text { ) }\end{array}$ & $\begin{array}{l}\mathrm{Bi}(20) \\
\mathrm{Fe}(20 \\
\mathrm{O}(60)\end{array}$ & $\begin{array}{l}\mathrm{Bi}(18) \\
\mathrm{Gd}(2) \\
\mathrm{Fe}(20) \\
\mathrm{O}(60)\end{array}$ & $\begin{array}{l}\mathrm{Bi}(18) \\
\mathrm{Gd}(2) \\
\mathrm{Fe}(19.2) \\
\mathrm{Ti}(0.2) \\
\mathrm{O}(60)\end{array}$ & $\begin{array}{l}\mathrm{Bi}(18) \\
\mathrm{Gd}(2) \\
\mathrm{Fe}(19) \\
\mathrm{Ti}(1) \\
\mathrm{O}(60)\end{array}$ & $\begin{array}{l}\text { Bi (18) } \\
\text { Gd (2) } \\
\text { Fe (18) } \\
\text { Ti (2) } \\
\text { O (60) }\end{array}$ \\
\hline Phases present (wt\%) & $\begin{array}{l}\mathrm{BFO}(82.8), \\
\mathrm{Bi}_{2} \mathrm{Fe}_{4} \mathrm{O}_{9}(15), \\
\mathrm{Bi}_{2} \mathrm{O}_{3}(1.2) \\
\alpha-\mathrm{Fe}_{2} \mathrm{O}_{3}(1)\end{array}$ & $\begin{array}{l}\mathrm{BGF}(92), \\
\mathrm{BiFeO}_{3}(5)-p b n m, \\
\mathrm{Bi}_{2} \mathrm{Fe}_{4} \mathrm{O}_{9}(2), \\
\mathrm{Bi}_{2} \mathrm{O}_{2.3}(1)\end{array}$ & $\begin{array}{l}\mathrm{BGFT}_{1}(95), \\
\mathrm{BiFeO}_{3}(5)-\text { pbnm }\end{array}$ & $\begin{array}{l}\mathrm{BGFT}_{2}(97), \\
\mathrm{Bi}_{2} \mathrm{O}_{2.5}(2), \\
\mathrm{Bi}_{2} \mathrm{O}_{2.3}\end{array}$ & $\begin{array}{l}\text { BGFT3 (98), } \\
\mathrm{Bi}_{2} \mathrm{O}_{2.5}(1), \\
\mathrm{Bi}_{2} \mathrm{O}_{2.3}\end{array}$ \\
\hline $\begin{array}{l}\text { Elemental composition } \\
\text { obtained from XRD (at } \%)\end{array}$ & $\begin{array}{l}\mathrm{Bi}(19.6), \\
\mathrm{Fe}(20), \\
\mathrm{O}(59.8)\end{array}$ & $\begin{array}{l}\text { Bi (18.71), } \\
\text { Gd (1.86), } \\
\text { Fe (20), } \\
\text { O (59.43) }\end{array}$ & $\begin{array}{l}\text { Bi (18.1), } \\
\text { Gd (1.9), } \\
\text { Fe (19.8), } \\
\text { Ti (0.2), } \\
\text { O (60) }\end{array}$ & $\begin{array}{l}\text { Bi (18.47), } \\
\text { Gd (1.98), } \\
\text { Fe (18.77), } \\
\text { Ti (0.98), } \\
\text { O (59.8) }\end{array}$ & $\begin{array}{l}\text { Bi (18.46), } \\
\text { Gd (1.96), } \\
\text { Fe (17.74), } \\
\text { Ti (1.96), } \\
\text { O (59.88) }\end{array}$ \\
\hline $\begin{array}{l}\text { Elemental composition } \\
\text { obtained from EDX (at } \%)\end{array}$ & $\begin{array}{l}\mathrm{Bi}(17.23), \\
\mathrm{Fe}(16.4), \\
\mathrm{O}(66.37)\end{array}$ & $\begin{array}{l}\text { Bi (16.31), } \\
\text { Gd (1.62), } \\
\text { Fe (16.24), } \\
\text { O (65.83) }\end{array}$ & $\begin{array}{l}\mathrm{Bi}(14.3), \\
\mathrm{Gd}(2.3), \\
\mathrm{Fe}(14.55), \\
\mathrm{Ti}(0.15), \\
\mathrm{O}(68.7)\end{array}$ & $\begin{array}{l}\text { Bi (15.2), } \\
\text { Gd (2.3), } \\
\text { Fe }(13.5), \\
\text { Ti }(0.3), \\
\text { O }(68.7)\end{array}$ & $\begin{array}{l}\text { Bi (14.3), } \\
\text { Gd (1.5), } \\
\text { Fe (10.5), } \\
\text { Ti (1.8), } \\
\text { O (71.9) }\end{array}$ \\
\hline$\rho_{\exp }\left(\mathrm{g} \mathrm{cc}^{-1}\right)$ & 7.69 & 7.84 & 7.58 & 7.80 & 7.56 \\
\hline$\rho_{\text {theo }}\left(\mathrm{g} \mathrm{cc}^{-1}\right)$ & 8.10 & 8.20 & 8.19 & 8.18 & 8.18 \\
\hline Densification (\%) & 94.93 & 95.56 & 92.58 & 95.29 & 92.38 \\
\hline
\end{tabular}

and cell volume etc., for the intended $\mathrm{BiFeO}_{3}$ major phase are listed in table 1 . The impurity phases present and their amounts in wt\% obtained from Rietveld refinement are listed in table 2.

Apart from the rhombohedral $\mathrm{Bi}_{0.9} \mathrm{Gd}_{0.1} \mathrm{FeO}_{3}$ phase (93 wt\%), the Rietveld refinement of XRD pattern of the BGF sample shows the presence of orthorhombic centrosymmetric $(P b n m) \mathrm{BiFeO}_{3}$ phase $(\sim 1 \mathrm{wt} \%)$ with lattice parameters $a=5.6083 \AA, b=7.703 \AA, c=5.3729 \AA$. It is worth mentioning that the existence of centrosymmetric (Pbnm) non-ferroelectric phase of $\mathrm{BiFeO}_{3}$ has been observed at high pressure [22]. About 2 wt $\%$ of $\mathrm{Bi}_{2} \mathrm{Fe}_{4} \mathrm{O}_{9}$ phase and $\sim 1 \mathrm{wt} \%$ of $\mathrm{Bi}_{2} \mathrm{O}_{2.3}(14 / \mathrm{mmm})$ are also present. Owing to the smaller ionic size of $\mathrm{Gd}(0.938 \AA)$ than that of $\mathrm{Bi}$ (1.31 $\AA$ ) [23], the lattice parameters of $\mathrm{Bi}_{0.9} \mathrm{Gd}_{0.1} \mathrm{FeO}_{3}$ phase are found to be less when compared to that of the undoped $\mathrm{BiFeO}_{3}$ phase (table 1). $\mathrm{Gd}^{3+}$ doping helped in reducing the $\mathrm{Bi}_{2} \mathrm{Fe}_{4} \mathrm{O}_{9}$ impurity phase formation from $\sim 15 \mathrm{wt} \%$ (present in undoped BFO) to $2 \mathrm{wt} \%$ (figure $1 \mathrm{~b}$ ). A similar observation was made earlier [24]. To determine the positions of $\mathrm{Gd}$ in the rhombohedral lattice of $\mathrm{BiFeO}_{3}$, we calculated the diffraction intensities by substituting $\mathrm{Gd}$ at the $\mathrm{Bi}$ - and 
Fe-sites separately. According to the Rietveld refinement, the best fit was obtained when Gd was substituted at the Bi site. It is understood that to stabilize the rhombohedral structure of $\mathrm{BiFeO}_{3}$, replacement of $\mathrm{Gd}$ at the $\mathrm{Bi}$-site plays an important role, as this site otherwise would have been vacant due to the volatile nature of $\mathrm{Bi}_{2} \mathrm{O}_{3}$ leading to the formation of Bi-deficient impurity phase (like $\mathrm{Bi}_{2} \mathrm{Fe}_{4} \mathrm{O}_{9}$ ). This impurity phase appears to be the source for higher conductivity/poor dielectric behaviour observed in $\mathrm{BFO}$ sample, in comparison with the theoretical estimation of dielectric behaviour for a monophasic $\mathrm{BiFeO}_{3}$ [25]. The formation of $\mathrm{Bi}_{2} \mathrm{Fe}_{4} \mathrm{O}_{9}$ phase due to $\mathrm{Bi}$ volatility is shown in the following reaction which takes place during the synthesis of $\mathrm{BiFeO}_{3}$ :

$4 \mathrm{Bi}_{2} \mathrm{O}_{3}+4 \mathrm{Fe}_{2} \mathrm{O}_{3} \rightarrow(2-x) 4 \mathrm{BiFeO}_{3}+(x) \mathrm{Bi}_{2} \mathrm{Fe}_{4} \mathrm{O}_{9}+(x) \mathrm{Bi}_{2} \mathrm{O}_{3} \uparrow$.

It should be noted that the above reaction occurs at a certain temperature. The presence of small amount of $\mathrm{Bi}_{2} \mathrm{O}_{3}$ phase is believed to be due to the fact that the released volatile $\mathrm{Bi}_{2} \mathrm{O}_{3}$ could not escape from the densely-pressed pellets and was trapped when cooled down to RT.

Refinements of XRD patterns of Gd and Ti co-doped samples (figure 1c-e) show the presence of $\sim 5 \mathrm{wt} \% \mathrm{BiFeO}_{3}$ (pbnm) phase for BGFT1; 2 $\mathrm{wt} \% \mathrm{Bi}_{2} \mathrm{O}_{2.5}\left(P-42{ }_{1} c\right)$, and $\sim 1 \mathrm{wt} \% \mathrm{Bi}_{2} \mathrm{O}_{2.3}(\mathrm{I} / \mathrm{mmm})$ for BGFT2; and $\sim 1 \mathrm{wt} \% \mathrm{Bi}_{2} \mathrm{O}_{2.5}$ $\left(P-42_{1} c\right), \sim 1 \mathrm{wt} \% \mathrm{Bi}_{2} \mathrm{O}_{2.3}(\mathrm{I} / \mathrm{mmm})$ phase for BGFT3. $\mathrm{X}$-ray diffraction studies suggest that the composition used for BGFT1 is best suited to obtain a single phase compound. However, Mössbauer studies carried out on BGFT1 samples indicate the presence of a small amount of $\mathrm{Bi}_{2} \mathrm{Fe}_{4} \mathrm{O}_{9}$ phase. Furthermore, the Rietveld refinement results confirm that $\mathrm{Ti}$ occupies the Fe-site. No significant variations in lattice parameters and the cell volumes were observed in Ti codoped samples with the variation of the dopant concentration (table 1).

The crystallite sizes and the associated strain for all the samples were calculated by using Williamson-Hall (W-H) method. There is a decreasing trend in crystallite size on increasing the dopant concentration. An increasing trend in strain from the undoped samples to the doped samples was observed and is elucidated in table 1 . However, there is a rapid fall in strain and crystallite size for BGFT3. The reason for this could be due to higher sintering temperature involved in obtaining dense $(92.38 \%)$ ceramics. These strains are originating from the differences in ionic radii and valence of dopants, $\operatorname{Gd}(0.938 \AA)$ and $\mathrm{Ti}(0.605 \AA)$ with respect to host atoms $\mathrm{Bi}(1.31 \AA)$ and $\mathrm{Fe}(0.645 \AA)$. The strain is believed to have played an important role in inhibiting the growth of crystallites with increasing dopant concentration. Furthermore, refinement of atomic coordinates and occupancy of atoms indicate the presence of dopants on preferred lattice sites (i.e., $\mathrm{Gd}$ at $\mathrm{Bi}$ and $\mathrm{Ti}$ at $\mathrm{Fe}$ sites, respectively).

3.1b Mössbauer spectroscopy: Mössbauer spectra obtained for all samples under investigation are shown in figure $2 \mathrm{a}-\mathrm{e}$. The spectra were fit using the 'Normos' computer program

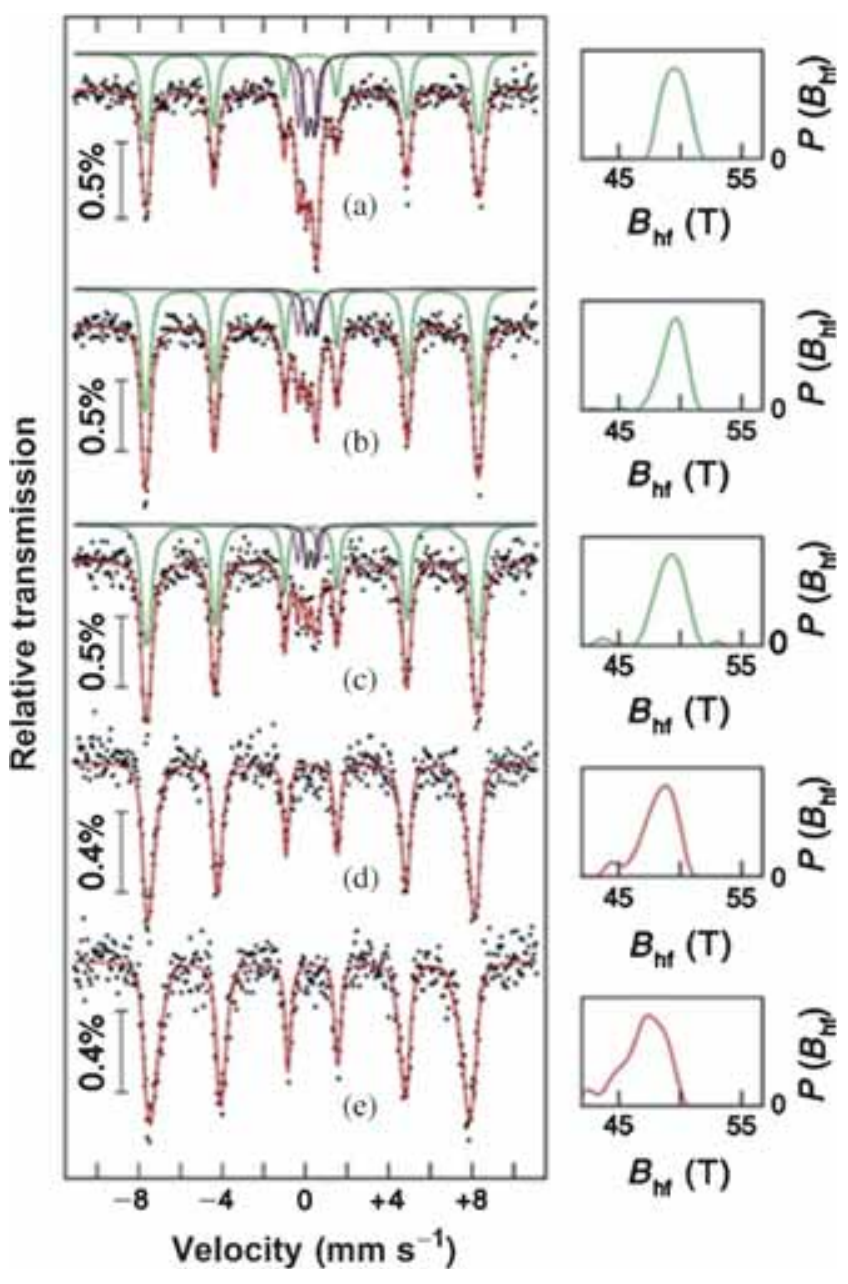

Figure 2. Room temperature ${ }^{57} \mathrm{Fe}$ Mössbauer spectra of the studied samples: (a) BFO, (b) BGF, (c) BGFT1, (d) BGFT2 and (e) BGFT3 samples. The circles represent the measured data and the solid lines are least square fittings to the measured data. The probability distribution of magnetic hyperfine field $\left\langle B_{\mathrm{hf}}\right\rangle$ is shown on the right hand side of the figure.

[26]. The parameters obtained from the least-square fitting are given in table 3. All isomer shifts (IS) are given relative to the ${ }^{57} \mathrm{Co}(\mathrm{Rh})$ source, which produces an IS of $-0.106 \mathrm{~mm} \mathrm{~s}^{-1}$ in $\alpha$-Fe foil at RT.

Mössbauer spectrum of BFO sample is shown in figure 2a. The spectrum consists of a large sextet and two central doublets. The sextet is non-symmetrical with respect to the zero velocity position and the spectral line width for the sextet is much larger than the natural line width. Hence, the spectrum was fit with a distribution of sextets and two central doublets D1 and D2. The obtained average hyperfine field for the sextet $\left\langle B_{\mathrm{hf}}\right\rangle=49.5( \pm 0.2)$ Tesla (T) and IS of $0.281 \mathrm{~mm} \mathrm{~s}^{-1}$ are typical for $\mathrm{BiFeO}_{3}$ [26]. Hence, this sextet is unambiguously assigned to the undoped $\mathrm{BiFeO}_{3}$ phase. The sextet distribution suggests that the position of $\mathrm{Fe}$ in the crystal lattice is not unique, but slightly varying, which might be due to the spin cycloid formation in $\mathrm{BiFeO}_{3}$ [7]. The central part of the spectrum is fit with two quadrupole doublets. We assign this 
Table 3. ${ }^{57} \mathrm{Fe}$ hyperfine parameters obtained from the fitting of Mössbauer spectra of BFO, BGF, BGFT1, BGFT2 and BGFT3 samples. IS (isomer shift), $B_{\mathrm{hf}}$ (magnetic hyperfine field in Tesla) and QS (quadrupole splitting). The isomer shift values are with respect to the ${ }^{57}$ Co source in Rh-matrix. The sextet and the doublets D1 and D2 are as shown in figure 3. Skewness is related to the hyperfine field distribution given on the right hand side of the Mössbauer spectra in figure 2.

\begin{tabular}{|c|c|c|c|c|c|c|}
\hline Samples & & $\begin{array}{l}\text { Relative area } \\
(\%)( \pm 1)\end{array}$ & Skewness & $\begin{array}{c}\text { IS }\left(\mathrm{mm} \mathrm{s}^{-1}\right) \\
\quad( \pm 0.005)\end{array}$ & $\begin{array}{c}\mathrm{QS}\left(\mathrm{mm} \mathrm{s}^{-1}\right) \\
\quad( \pm 0.05)\end{array}$ & $\begin{array}{l}\left\langle B_{\mathrm{hf}}\right\rangle(\mathrm{T}) \\
( \pm 0.2 \mathrm{~T})\end{array}$ \\
\hline \multirow[t]{3}{*}{$\mathrm{BFO}$} & Sextet & 62.39 & \multirow[t]{3}{*}{-1.06} & 0.18 & 0.11 & \multirow[t]{3}{*}{49.52} \\
\hline & D1 & 18.1 & & 0.13 & 0.933 & \\
\hline & D2 & 19.51 & & 0.24 & 0.387 & \\
\hline \multirow[t]{3}{*}{ BGF } & Sextet & 80.3 & \multirow[t]{3}{*}{-0.869} & 0.21 & 0.056 & \multirow[t]{3}{*}{49.41} \\
\hline & D1 & 10.15 & & -0.11 & -0.903 & \\
\hline & D2 & 9.541 & & 0.27 & 0.406 & \\
\hline \multirow[t]{3}{*}{ BGFT1 } & Sextet & 85.46 & \multirow[t]{3}{*}{-1.33} & 0.248 & 0.060 & \multirow[t]{3}{*}{49.18} \\
\hline & D1 & 7.065 & & 0.128 & 1.011 & \\
\hline & D2 & 7.477 & & 0.248 & 0.375 & \\
\hline BGFT2 & Sextet & 95.67 & -0.729 & 0.259 & -0.018 & 48.37 \\
\hline BGFT3 & Sextet & 100 & -0.724 & 0.271 & 0.001 & 47.3 \\
\hline
\end{tabular}

part of the spectrum to the unwanted phase formed during the synthesis of the sample (solid-state sintering) due to $\mathrm{Bi}_{2} \mathrm{O}_{3}$ volatility. The IS $=0.4 \mathrm{~mm} \mathrm{~s}^{-1}$, quadrupole shift $(\mathrm{QS})=0.8$ $\mathrm{mm} \mathrm{s}^{-1}$ for doublet D1, and IS $=0.6 \mathrm{~mm} \mathrm{~s}^{-1}$, QS $=0.4$ $\mathrm{mm} \mathrm{s}^{-1}$ for doublet D2 are matching well to the IS and QS of $\mathrm{Fe}$ atom at the tetrahedral and octahedral $\mathrm{Fe}$-sites of the orthorhombic $\mathrm{Bi}_{2} \mathrm{Fe}_{4} \mathrm{O}_{9}$ phase, respectively $[27,28]$. Hence from these subspectra, the impurity phase is identified to be $\mathrm{Bi}_{2} \mathrm{Fe}_{4} \mathrm{O}_{9}$.

The area under (both) the doublets to that of the sextets distribution is a measure of the $\mathrm{Fe}$ atoms present in the impurity phase $\left(\mathrm{Bi}_{2} \mathrm{Fe}_{4} \mathrm{O}_{9}\right)$ relative to that of the pure $\mathrm{BiFeO}_{3}$ phase. The spectral area of $\sim 38 \%$ (for both the doublets) accounts for the presence of $\mathrm{Bi}_{2} \mathrm{Fe}_{4} \mathrm{O}_{9}$ impurity phase and $\sim 62 \%$ for the pure $\mathrm{BiFeO}_{3}$ phase. A simple calculation estimates about $23 \mathrm{wt} \%$ of $\mathrm{Bi}_{2} \mathrm{Fe}_{4} \mathrm{O}_{9}$ present in the $\mathrm{BFO}$ sample. It is to be noted that $\sim 1 \mathrm{wt} \%$ of $\alpha-\mathrm{Fe}_{2} \mathrm{O}_{3}$ is present in $\mathrm{BFO}$ sample based on Rietveld analysis of the XRD data. However, the Mössbauer spectra corresponding to $\mathrm{BiFeO}_{3}$ did not reveal the presence of this phase as this could be due to the overlap between the sextets of $\mathrm{BiFeO}_{3}$ and $\alpha-\mathrm{Fe}_{2} \mathrm{O}_{3}$. Hence, the amount of $\alpha-\mathrm{Fe}_{2} \mathrm{O}_{3}$ present, could not be quantified by Mössbauer studies. The discrepancy that exists between the XRD and Mössbauer data, on the amount of $\mathrm{Bi}_{2} \mathrm{Fe}_{4} \mathrm{O}_{9}$ phase present, is attributed to its partial amorphous nature. The centrosymmetric orthorhombic $\mathrm{BiFeO}_{3}, \sim 1 \mathrm{wt} \%$ (observed in XRD) could not be observed by Mössbauer spectroscopy due to the high signal to noise ratio.

For the BGF sample, the recorded Mössbauer spectrum is shown in figure $2 \mathrm{~b}$. The spectrum is similar to that obtained for the BFO sample and was also fit using the same model. From the IS and QS values of the central doublets, it is evident that this sample also contains $\mathrm{Bi}_{2} \mathrm{Fe}_{4} \mathrm{O}_{9}$ as an impurity phase. The total area under the sextet and the two doublets are 80 and $20 \%$, respectively. The relative total area of both central doublets is low, i.e., the relative spectral area is $20 \%$, against $38 \%$ for the BFO sample. Hence, it is concluded that the amount of impurity phase is reduced from
23 to $13 \mathrm{wt} \%$. This result indeed confirms that Gd-doping facilitate a reduction in the amount of $\mathrm{Bi}_{2} \mathrm{Fe}_{4} \mathrm{O}_{9}$ impurity.

The observed average magnetic hyperfine field $\left\langle\mathrm{B}_{\mathrm{hf}}\right\rangle=$ $49.4( \pm 0.2) \mathrm{T}$ is not different from that of $49.5( \pm 0.2) \mathrm{T}$ observed for BFO sample (table 3). This indicates that the incorporation of $\mathrm{Gd}$ at the $\mathrm{Bi}$ site of $\mathrm{BiFeO}_{3}$ structure does not significantly affect the magnetic moment of Fe. This substitution of $\mathrm{Gd}$ at the Bi site is in agreement with the Rietveld analysis of the XRD patterns (discussed in the previous section). The other interesting point is that the incorporation of $\mathrm{Gd}$ stabilizes the rhombohedral (ferroelectric) $\mathrm{BiFeO}_{3}$ phase, which is inferred from sharpening of the $P\left(B_{\mathrm{hf}}\right)$ profile in figure $2 \mathrm{~b}$ right (as compared to figure $2 \mathrm{a}$, right). It is worth mentioning that the amount of $\mathrm{Bi}_{2} \mathrm{Fe}_{4} \mathrm{O}_{9}$ phase, as observed by $\mathrm{XRD}$, is very small $(\sim 2 \mathrm{wt} \%)$ in comparison with $\sim 13$ wt $\%$ (as observed by Mössbauer spectroscopy) suggesting that this impurity phase is mostly present in amorphous form.

The Mössbauer spectrum corresponding to the Gd- and Tico-doped (BGFT1) sample with $0.01 \mathrm{~mol} \%(x=0.01)$ Tidoping at the $\mathrm{Fe}$ site is shown in figure $2 \mathrm{c}$ and is fit using the similar model as in the case of the above two samples. Although, we have not observed the presence of $\mathrm{Bi}_{2} \mathrm{Fe}_{4} \mathrm{O}_{9}$ impurity phase in the XRD pattern (as it could be in amorphous state), Mössbauer spectrum reveals the presence of $\sim 6 \mathrm{wt} \%$ of this phase, which establishes the sensitivity of the Mössbauer spectroscopy towards detection of the Fecontaining impurity phases in $\mathrm{BiFeO}_{3}$ samples. The observed average value of $\left\langle B_{\mathrm{hf}}\right\rangle$ decreases to $49.0 \mathrm{~T}$. This larger reduction of $\left\langle B_{\mathrm{hf}}\right\rangle$ suggests the incorporation of $\mathrm{Ti}$ at the Fe site. Furthermore, the distribution of $\mathrm{Ti}$ ions at the Fe site makes the $\left\langle B_{\mathrm{hf}}\right\rangle$ distribution $P\left(B_{\mathrm{hf}}\right)$ broader in comparison with that of the BFO and BGF samples. The skewness of hyperfine field distribution given in table 3 represents the change in average $B_{\mathrm{hf}}$ due to Gd- and Ti-doping. As seen, the negative skewness represents the fact that Ti doping reduces $\left\langle B_{\mathrm{hf}}\right\rangle$ of the $\mathrm{Fe}$ atoms by reducing the number of $\mathrm{Fe}$ atoms around the core Fe-atom. 
Interestingly, the $x=0.05$ and 0.1 Ti-doped samples show no signature of any impurity phases as confirmed by both the XRD (figure 1d and e, respectively) and Mössbauer (figure 2d and e) studies. As described for the BGFT1 sample, the distribution of nonmagnetic $\mathrm{Ti}$ atoms substituted at the $\mathrm{Fe}$ site of $\mathrm{BiFeO}_{3}$ structure, decreases the average value of the magnetic hyperfine field $\left\langle B_{\mathrm{hf}}\right\rangle$ to 48.2 and $47.2 \mathrm{~T}$, respectively, for BGFT2 and BGFT3 samples. Besides the decrease in $\left\langle B_{\mathrm{hf}}\right\rangle$, we have also observed a broadening of the $\left\langle B_{\mathrm{hf}}\right\rangle$ distribution $\left(P\left(B_{\mathrm{hf}}\right)\right)$, which agree very well with the results obtained by Rietveld analysis of the XRD patterns. This is ascribed to an increase in strain with an increase in dopant concentration.

The results from the analysis of the Mössbauer spectra show that the amount of impurity phase in $\mathrm{BiFeO}_{3}$ can be controlled/eliminated by doping with suitable dopant ions ( $\mathrm{Gd}$ at the Bi-site and $\mathrm{Ti}$ at the $\mathrm{Fe}$-site) in appropriate concentration. The impurity phase that was generally found to form during the solid-state synthesis of $\mathrm{BiFeO}_{3}$ sample was identified to be $\mathrm{Bi}_{2} \mathrm{Fe}_{4} \mathrm{O}_{9}$. Mössbauer spectroscopy is proved to be very sensitive to detect all the Fe-containing impurity (in amorphous state), which otherwise could not be observed by XRD studies. Ti-doping reduces the average magnetic hyperfine field of $\mathrm{BiFeO}_{3}$ phase drastically as seen in table 3 . This indicates that the nonmagnetic $\mathrm{Ti}^{4+}$ ions reduce net Fe-magnetic moment by changing the Fe environment. Furthermore, according to the magnetometry studies discussed later, Ti-doping breaks the antiferromagnetic spin ordering that results in enhancing the magnetic properties of the samples. Hence, it is clear that co-doping of Gd and Ti is essential for improving the characteristics of $\mathrm{BiFeO}_{3}$ ceramics, as Gd-doping compensates for the Bi-volatility by providing a stable phase and Ti-doping improves the dielectric property besides improving the ferromagnetic properties.

According to previous reports $[9,19]$, the oxygen vacancies were created in $\mathrm{BiFeO}_{3}$ samples due to Bi-volatility. To compensate the oxygen vacancies, $\mathrm{Fe}^{3+}$ transforms to $\mathrm{Fe}^{2+}$ to maintain the charge balance and the $\mathrm{BiFeO}_{3}$ becomes $\mathrm{Bi}\left(\mathrm{Fe}_{1-x}^{3+} \mathrm{Fe}_{x}^{2+}\right) \mathrm{O}_{3-x / 2}$. Creation of oxygen vacancy may be rationalized by the following equation (2):

$$
6 \mathrm{BiFeO}_{3} \rightarrow 2 \mathrm{Fe}_{3} \mathrm{O}_{4}+3 \mathrm{Bi}_{2} \mathrm{O}_{3}+\mathrm{O} \uparrow .
$$

However, the Mössbauer spectra for the entire series of the samples do not show the presence of $\mathrm{Fe}^{2+}$. Hence, from the Mössbauer results it is concluded that the high conductivity of, especially, the BFO sample is not directly related to the oxygen vacancy formation.

\subsection{Microstructural studies}

Figure 3 shows SEM images of representative samples: BFO, BGFT2 and BGFT3. The average crystallite sizes of BFO, BGF, BGFT1, BGFT2 and BGFT3 samples are 1.41, 1.21, $1.08,1.03$ and $0.236 \mu \mathrm{m}$, respectively. The change in crystallite size and morphology (from rectangular to nearly spherical) indicates that the Gd- and Ti-doping effects the crystallization/growth habit of BFO crystallites. The crystallites of BFO samples are large and mostly rectangular in shape.

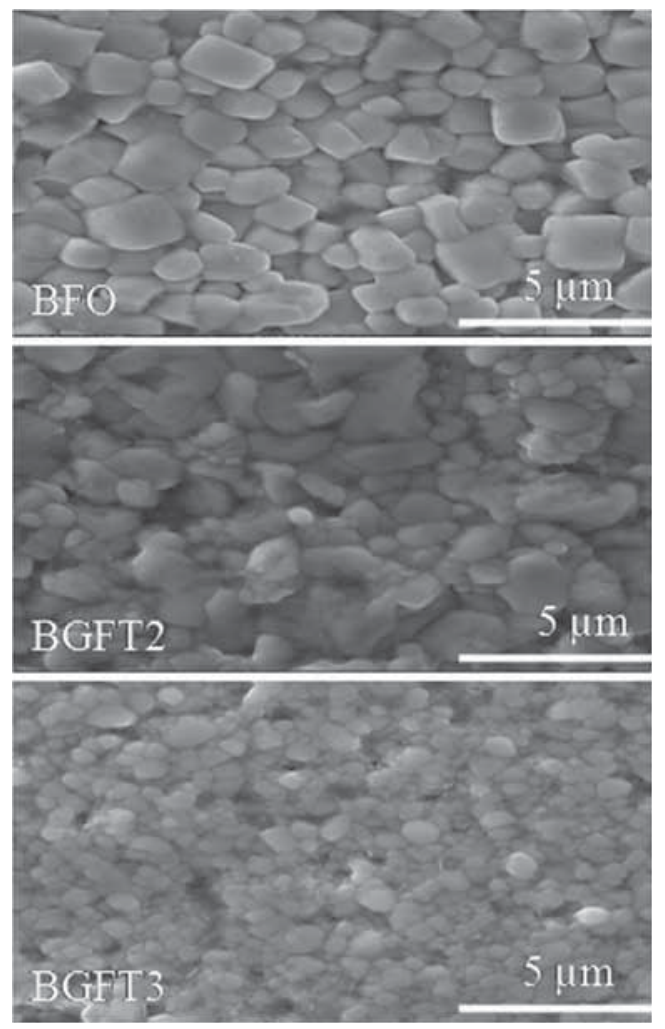

Figure 3. Scanning electron micrographs of BFO, BGFT2 and BGFT3 samples.

On Gd-doping, although, the shape of the crystallites seems to be identical with that of BFO samples, their average size reduces. For samples with increase in Ti-doping a nonuniform morphology having smaller sizes has been observed. The smaller grain size is owing to the strain produced by the dopant ions that are smaller in size as corroborated by lattice shrinkage (table 1).

The SEM images (figure 3) indicate that increasing the amount of Ti-doping significantly decreases the grain size of BFO ceramics. Due to the higher valence of $\mathrm{Ti}^{4+}$ than $\mathrm{Fe}^{3+}$, it plays a crucial role as an electron donor to the oxygen ions, thereby suppressing the formation of oxygen vacancies. This results in slower oxygen-ion motion and subsequently lower rate of grain growth [29].

\subsection{Chemical composition by energy dispersive X-ray (EDX) spectroscopy}

To estimate the amount of impurity phases present and loss of $\mathrm{Bi}$ from the sample due to its volatility, we have performed EDX analysis. The results obtained are illustrated in table 2. The elemental composition of each sample was also estimated from the X-ray diffraction results, considering the weight percent of the phase present. These results are also listed in table 2. For the BFO and BGF samples, it is clear that the composition of $\mathrm{Bi}$ is about 2 and $1 \mathrm{wt} \%$, respectively, i.e., less than the stoichiometric composition. However, no Bi-loss was seen for the other samples, i.e., BGFT1, BGFT2 and BGFT3. Hence, it is inferred that the 
formation of $\mathrm{BiFeO}_{3}$ phase, with both $\mathrm{Bi}^{3+}$ and $\mathrm{Fe}^{3+}$ cations being in $3+$ valency $\left(\mathrm{Bi}^{3+}, \mathrm{Fe}^{3+}\right)$ state, is not favoured. However, the growth of the rhombohedral phase is more facilitated by different valence ions like $\mathrm{Ti}^{4+}$.

\subsection{Ferroelectric properties}

For making ferroelectric measurements we used the pellets of BFO, BGF, BGFT1, BGFT2 and BGFT3 samples obtained by solid-state sintering process. Often, the density of the pellets used for ferroelectric measurements plays an important role as it depends on the presence of voids and grain boundaries. Hence, it is important to determine the densities of the pellets for better understanding of the dielectric and ferroelectric properties.

Theoretical densities of all the samples were calculated by averaging over the densities of all the phases present in respective samples. For example, according to the Rietveld refinement results, the phases present in $\mathrm{BFO}$ sample are $\mathrm{BiFeO}_{3}(82.47 \mathrm{wt} \%), \mathrm{BiFeO}_{3}($ Pnma $\sim 1 \mathrm{wt} \%), \mathrm{Bi}_{2} \mathrm{Fe}_{4} \mathrm{O}_{9}$ ( 15 wt $\%), \alpha-\mathrm{Fe}_{2} \mathrm{O}_{3}(\sim 1 \mathrm{wt} \%)$ and $\mathrm{Bi}_{2} \mathrm{O}_{3}(\sim 1 \mathrm{wt} \%)$. The densities of $\mathrm{BiFeO}_{3}\left(8.33 \mathrm{~g} \mathrm{cc}^{-1}\right)$ [30], $\mathrm{Bi}_{2} \mathrm{Fe}_{4} \mathrm{O}_{9}$ (7.14 g $\left.\mathrm{cc}^{-1}\right)$ [31], $\alpha-\mathrm{Fe}_{2} \mathrm{O}_{3}\left(5.24 \mathrm{~g} \mathrm{cc}^{-1}\right)$ and $\mathrm{Bi}_{2} \mathrm{O}_{3}\left(8.90 \mathrm{~g} \mathrm{cc}^{-1}\right)$ [32] were considered for calculation of average theoretical density (without accounting for porosity in various phases), which results in a theoretical density of $8.10 \mathrm{~g} \mathrm{cc}^{-1}$ for BFO sample. A density of $7.69 \mathrm{~g} \mathrm{cc}^{-1}$ was obtained experimentally for the BFO sample by using Archimedes principle. For all the samples studied, the theoretical and experimental densities were obtained by the same method as explained above and are listed in table 2. In the case of BGF, BGFT1, BGFT2 and BGFT3 samples, the densities of Gd- and Ti-substituted $\mathrm{BiFeO}_{3}$-phases were calculated by replacing the respective atoms at their respective sites in the $\mathrm{BiFeO}_{3}$ structure by keeping the unit cell volume constant. For example, density of $\mathrm{Bi}_{0.9} \mathrm{Gd}_{0.1} \mathrm{Fe}_{0.9} \mathrm{Ti}_{0.1} \mathrm{O}_{3}$ was determined by calculating its formula-weight and comparing with the weight of one formula unit of $\mathrm{BiFeO}_{3}$, assuming the cell volume to be constant. As there is almost no change in cell volume observed by XRD, calculating density using this method would involve minimal error. The calculated densities obtained for $\mathrm{BiFeO}_{3}$ (BFO), BGF, BGFT1, BGFT2 and BGFT3 phases were 8.10, $8.2,8.19,8.18$ and $8.18 \mathrm{~g} \mathrm{cc}^{-1}$, respectively. The experimental and theoretical densities of all the analysed samples are listed in table 2.

Regarding the remnant polarization associated with these samples, the conventional Sawyer-Tower method (Radiant Technology) was employed at RT [33]. Figure 4 shows the polarization $v s$. electric field $(P-E)$ hysteresis loop obtained for the representative BGFT2 sample at the frequency of $100 \mathrm{~Hz}$ and at applied field of $10 \mathrm{kV} \mathrm{cm}^{-1}$. In BFO, the displacement of $\mathrm{Bi}$ is responsible for the polarization (displacement of $\mathrm{Bi}$ is three times than that of $\mathrm{Fe}$ ) [34]. This indicates that the polarization of $\mathrm{Bi}$ is three times than that of $\mathrm{Fe}$. As shown in figure 4 , the BGFT2 sample exhibited $P$ vs. $E$ hysteresis loop, which is not well saturated. It seems that the leakage current due to the presence of impurities and amorphous-like phases are the important limiting parameters. Since the $P$ vs. $E$ loops are not saturated, quantifying the remanent and saturation polarization is difficult and the $P v s$. $E$ loops of all other samples are not included in figure 4 . The lower values of polarization associated with the samples are attributed to the presence of smaller grains.

We have observed that as the dopant ( $\mathrm{Gd}$ and $\mathrm{Ti}$ ) concentration increases in BFO, there is an increasing trend in polarization due to the reduction of impurity in BFO ceramics apart from the distortion of rhombohedral $R 3 c$ structure. The distortion in the $\mathrm{ABO}_{3}$ perovskite leads to the displacement of ions which results in an increase in dipole moment and thus, the polarization increases. The undoped BFO sample showed lower polarization value as a consequence of the formation of $\mathrm{Bi}_{2} \mathrm{Fe}_{4} \mathrm{O}_{9}$ phase having high electrical conductivity. Donor dopant $\mathrm{Ti}^{4+}$ on Fe sites resulted in a decrease impurity phase content and hence, an improvement in the polarization value. Doping may reduce the oxygen-related defects which reduces the leakage current thereby improving the pinning defects and enhancing the polarization [17]. Further, under the application of largest voltage $(>15 \mathrm{kV}$ $\mathrm{cm}^{-1}$ ) possible in our measurement system, the sample did not break dielectrically. However, the absence of saturation in $P$ vs. $E$ loops suggests higher coercive fields associated with these samples at RT.

\subsection{Magnetic properties}

Figure 5 shows magnetization hysteresis (M-H) loops obtained at RT with an applied field upto 2 Tesla (T) for BFO, BGF, BGFT1, BGFT2 and BGFT3 samples. The paramagnetic contribution has been subtracted for better estimation of the saturation magnetization. The observed values of remnant magnetization $\left(M_{\mathrm{r}}\right)$ of BFO, BGF, BGFT1, BGFT2 and BGFT3 samples are 0.002, 0.020, 0.037, 0.05 and 0.237 emu $\mathrm{g}^{-1}$, respectively, and the corresponding

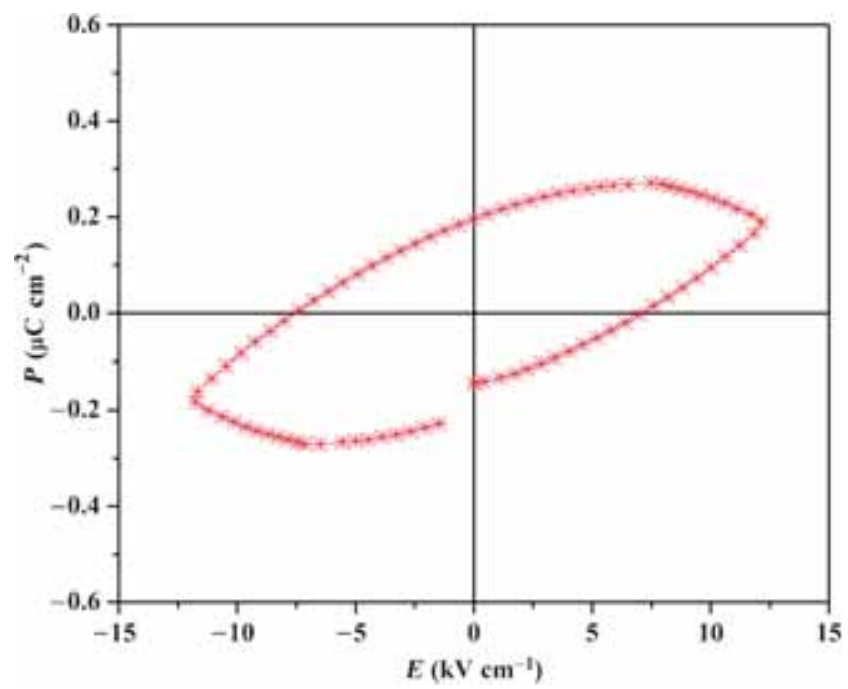

Figure 4. Ferroelectric hysteresis loops at RT, the frequency of $100 \mathrm{~Hz}$ and at the applied field of $1000 \mathrm{~V}$ for BGFT2 sample. 


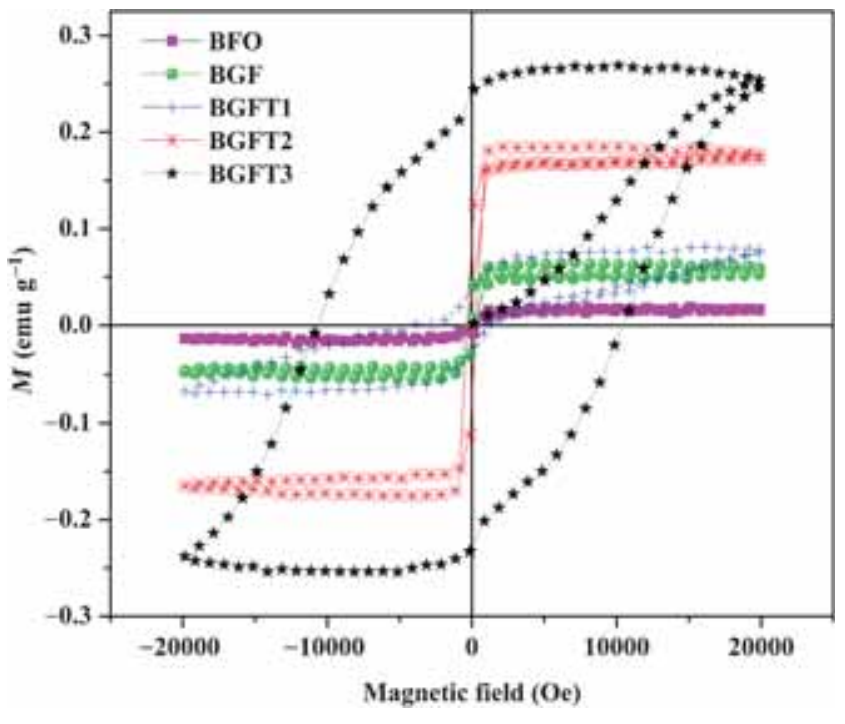

Figure 5. Magnetic hysteresis loops at RT for BFO, BGF, BGFT1, BGFT2 and BGFT3 samples.

Table 4. Remnant magnetization $\left(M_{\mathrm{r}}\right)$ and saturation magnetization for BFO, BGF, BGFT1, BGFT2 and BGFT3 samples.

\begin{tabular}{lcc}
\hline Samples & $M_{\mathrm{r}}\left(\mathrm{emu} \mathrm{g}^{-1}\right)$ & $M_{\mathrm{s}}\left(\mathrm{emu} \mathrm{g}^{-1}\right)$ \\
\hline BFO & 0.002 & 0.02 \\
BGF & 0.022 & 0.023 \\
BGFT1 & 0.04 & 0.07 \\
BGFT2 & 0.05 & 0.18 \\
\hline
\end{tabular}

saturation magnetization $\left(M_{\mathrm{s}}\right)$ values are $0.016,0.06,0.07$, 0.187 and $0.267 \mathrm{emu} \mathrm{g}^{-1}$, respectively (table 4). The undoped BFO sample is antiferromagnetic in nature with negligibly small remnant and saturation magnetization, as the $\mathrm{BiFeO}_{3}$ phase is a G-type antiferromagnet with a cycloidal spin arrangement (having a period of $620 \AA$ ) [6]. The impu rity phases of $\mathrm{Bi}_{2} \mathrm{Fe}_{4} \mathrm{O}_{9}, \alpha-\mathrm{Fe}_{2} \mathrm{O}_{3}$ and $\mathrm{Bi}_{2} \mathrm{O}_{3}$ are paramagnetic, antiferromagnetic (from Mössbauer spectra, figure 2) and diamagnetic, respectively. Hence, they do not contribute to the net magnetization. The Gd-doped BFO ceramic sample (BGF) has a very small increased magnetic moment but is still antiferromagnetic-like. This small increase in magnetization is attributed to the contribution from $4 \mathrm{f} \mathrm{Gd}$-spins, and uncompensated $\mathrm{Fe}$-spins due to their small exchange interaction with Gd-spins. This behaviour supports the results of XRD and Mössbauer spectroscopy, Gd that occupies Bi site without altering the $\mathrm{Fe}$-spin arrangement in the structure. The substitution of both $\mathrm{Gd}$ and $\mathrm{Ti}$ in $\mathrm{BFO}$ ceramics increases the magnetic moment, and hence, the magnetization, as the concentration of $\mathrm{Ti}$ in the sample is increased. This further proves the substitution of $\mathrm{Ti}$ at the $\mathrm{Fe}$ site leaves uncompensated Fe-spins to contribute to the total magnetization. In other words, due to the suppression of antiferromagnetic spin structure of BFO $[15,35]$. BGFT3 has high saturation magnetization and shows improved ferromagnetic behaviour. Variation of saturation magnetization as a function of Ti doping suggests that magnetic property enhancement is caused more likely due to the formation of ferromagnetic and/or ferrimagnetic ordering rather than the grain size effect, as the grain size associated with the present samples are well above the critical size $(620 \AA)$ which is reported in the literature. It is clear from figure 5 that the coercivity of the samples becomes larger by doping with Ti. This may be due to the fact that although the antiferromagnetic spin-arrangement is broken by the nonmagnetic $\mathrm{Ti}$ ions, the Fe-spins are still strongly interacting with the neighbouring Fe-spins.

\section{Conclusions}

$\mathrm{BiFeO}_{3}$ and $\mathrm{Bi}_{0.9} \mathrm{Gd}_{0.1} \mathrm{Fe}_{1-x} \mathrm{Ti}_{x} \mathrm{O}_{3}(x=0,0.01,0.05$ and 0.1 ) were synthesized via solid-state reaction route. Rietveld analyses of the XRD patterns revealed the structure of $\mathrm{BiFeO}_{3}$ and $\mathrm{Bi}_{0.9} \mathrm{Gd}_{0.1} \mathrm{Fe}_{1-x} \mathrm{Ti}_{x} \mathrm{O}_{3}(x=0,0.01,0.05$ and 0.1 ) to be rhombohedral associated with $R 3 c$ space group. By Gd and Ti co-doping, the concentration of impurity phase was found to decrease. The quantitative estimation of phase fractions for all the samples obtained from analysis of the Mössbauer spectra agrees well with Rietveld analysis of XRD data. The increase in magnetization as observed in the magnetic hysteresis loop measured at RT by VSM, the observed ferroelectric and improved magnetic properties are well supported by the structural information obtained from the XRD and Mössbauer results. According to the variation of the $\left\langle B_{\mathrm{hf}}\right\rangle$ obtained from the analysis of Mössbauer spectra, Gd-doping at the Bi-site does not decrease the magnetic moment, because there is no modification involved in antiferromagnetic coupling of $\mathrm{Fe}$-spins in $\mathrm{BiFeO}_{3}$. However, by doping non-magnetic $\mathrm{Ti}$ ion at the $\mathrm{Fe}$ site, the antiferromagnetic Fe-spin alignment is broken and hence, increased magnetization was observed by VSM with increase in Ti-doping.

\section{Acknowledgement}

S Madolappa greatly acknowledges the University Grants Commission (UGC, New Delhi), Government of India, for awarding Dr D S Kothari, post-doctoral fellowship wide order no. F.4-2/2006 (BSR)/13-907/2013 (BSR).

\section{References}

[1] Rovillian P, Desouza R, Gallias Y, Sacuto A, Measson M A, Colson D et al 2010 Nature Mater. 9975

[2] Lazenka L V, Zhang G, Vanacken J, Makoed I I, Ravinsk A F and Moshchalkov V V 2012 J. Phys. D: Appl. Phys. 45 125002

[3] Fiebig M 2005 J. Phys. D: Appl. Phys. 38 R123

[4] Nan C W, Liu G, Lin Y-H and Chen H 2005 Phys. Rev. Lett. 94197203 
[5] Preethi Meher K R S and Varma K B R 2009 J. Appl. Phys. 106124103

[6] Spaldin N A, Cheong S and Ramesh R 2010 Physics Today 6338

[7] Kothari D, Reddy V R, Gupta A, Phase D M, Lakshmi N, Deshpande S K and Awasthi A M 2007 J. Phys.: Condens. Matter 19136202

[8] Catalan B G and Scott J F 2009 Adv. Mater. 212463

[9] Pradhan A K, Zhang K, Hunter D, Dadson J B, Louts G B, Bhattacharya P et al 2005 J. Appl. Phys. 97093903

[10] Kalantari K S, Sinclair D C, Bingham P A, Pokorny J and Raeney I M 2012 J. Appl. Phys. 111064107

[11] Agarwal R A, Ashima S S and Ahlawat N 2012 J. Phys. D: Appl. Phys. 45165001

[12] Ruette B, Zvyagin S, Pyatakov A P, Bush A, Li J F, Belotelov V I et al 2004 Phys. Rev. B 69064114

[13] Lin Y, Jiang Q, Wang Y and Nan C 2007 Appl. Phys. Lett. 90 172507

[14] Kumar M M, Srinath S, Kumar G S and Suryanarayana S V 1998 J. Magn. Magn. Mater. 188203

[15] Cheng Z X, Wang X L, Du Y and Dou S X 2010 J. Phys. D: Appl. Phys. 43242001

[16] Wang Y and Nan C W 2006 Appl. Phys. Lett. 89052903

[17] Lotey G S and Verma N K 2012 J. Nanopart Res. 14742

[18] Srinivas A, Kin D W, Hong K S and Suryanaryana S V 2003 Appl. Phys. Lett. 832217

[19] Basith M A, Kurni O, Alam M S, Sinha B L and Ahmmad B 2014 J. Appl. Phys. 115024102

[20] Kumar M, Sat P C and Chhoker S 2014 J. Mater. Sci: Mater. Electron. 255366
[21] Rodriguez-Carvajal J 1990 'FULLPROF: a program for Rietveld refinement and pattern matching analysis', Abstracts of the Satellite Meeting on powder diffraction of the XV Congress of the IUCr, p 127, Toulouse, France

[22] Arnold D C, Night K S, Morrison F D and Lightfoot P 2009 Phys. Rev. Lett. 102027602

[23] Shannon R D 1976 Acta Crystallogr. A 32751

[24] Lubk A, Gemming S and Spaldin N A 2009 Phys. Rev. B 80 104110

[25] Brand R A 1987 Nucl. Instrum. Methods B 28398

[26] Blaauw C and Van der Woulde F 1973 J. Phys. C: Solid State Phys. 61422

[27] Kostiner E and Shoemaker G L 1971 J. Solid State Chem. 3 186

[28] Grault D, Hervieu M, Nguyen N and Raveaou B 1988 J. Solid State Chem. 76248

[29] Chaodan Z, Jun Y, Duanming Z, Bin Y, Yunyi W, Longhai W et al 2007 Int. Ferroelectric. 9431

[30] Lebeugle D, Colson D, Forget A, Viret M, Bonville P, Marucco J F and Fusil S 2007 Phys. Rev. B 76 024116

[31] Friedrich A, Biehler J, Morgenroth W, Wiehl L, Winkler B, Hanfland M et al 2012 J. Phys.: Condens. Matter 24145401

[32] David R 2005 CRC Handbook of Chemistry and Physics Lide (ed) Internet version 2005, http://www.hbcpnetbase.com (Boca Raton, FL: CRC Press)

[33] Sawyer C B and Tower C H 1930 Phys. Rev. 35269

[34] Fuji K, Kato H, Omoto K, Yashima M, Chen J and Xing X 2013 Phys. Chem. Chem. Phys. 156779

[35] Basu S, Hossai S K M, Chakarvorthy D and Pal M 2011 Curr. Appl. Phys. 11976 